\title{
Finite-Time Synchronization of Chaos Gyros via Terminal Sliding Mode Control
}

\author{
R.P. Xu \\ College of Mathematics \\ Qingdao University \\ Qingdao, P.R. China \\ College of Information Science and Engineering \\ Ocean University of China \\ Qingdao, P.R. China \\ C.C. Gao \\ College of Information Science and Engineering \\ Ocean University of China \\ Qingdao, P.R. China
}

\author{
M.M. Gao \\ College of Mathematics \\ Qingdao University \\ Qingdao, P.R. China
}

\begin{abstract}
The problem of finite-time synchronization between two chaotic gyros with uncertain and disturbances is investigated. On the basis of a double power reaching law, a nonsingular terminal sliding mode control algorithm was proposed to restrain chattering and improve convergence speed of terminal mode control. First, a new nonsingular terminal sliding surface is introduced and its finite-time convergence to the equilibrium is proved. Then, a sliding mode controller is proposed based on a double power reaching law to force the trajectories of the synchronization error system onto the sliding surface and remain on it forever, and the finite-time synchronization conditions are obtained. Finally, simulation results are presented to illustrate the effectiveness of the design.
\end{abstract}

Keywords-chaos gyros; finite-time synchronization; nonsingular terminal sliding mode; double power reaching law

\section{INTRODUCTION}

Synchronization of the chaotic dynamical systems has gained a great deal of interest among researchers and engineers from variety of research fields in recent years [1-5]. In this line, many different methods have been applied theoretically and experimentally to synchronize chaotic systems, such as active control, adaptive control, linear control and sliding mode control [6-10].

The gyro is one of the most interesting dynamical systems. Gyros have found useful applications in optics, navigation, aeronautics and space engineering fields. The pioneering paper on the concept of chaotic motion in gyros was not presented until 1981. Chen [11] analyzed the dynamics of a symmetric gyro with linear plus-cubic damping subjected to a harmonic excitation. Lei [12] proposed the active control method to achieve complete synchronization between two identical chaotic gyros. Yan [13] addressed the problem of the chaotic gyros with fully unknown parameters using adaptive sliding mode control. Yau [14] has developed fuzzy controllers for synchronizing two uncertain chaotic gyros. So far, most of the existing results related to synchronization mainly focused on asymptotic synchronization. From a practical point of view, however, it is more valuable that the synchronization objective is realized in a finite time. Furthermore, the finite-time synchronization has demonstrated better robustness and disturbance rejection properties.

In this paper, we present a design scheme for the terminal sliding mode (TSM) control which can realize chaotic synchronization between two chaotic gyros with uncertain and disturbances in finite time.

\section{SYSTEM DESCRIPTION}

The dynamics of a symmetrical gyro with linear-plus-cubic

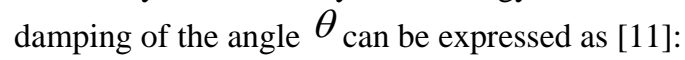

$$
\ddot{\theta}+c_{1} \dot{\theta}+c_{2} \dot{\theta}^{3}+\alpha^{2} \frac{(1-\cos \theta)^{2}}{\sin ^{3} \theta}-\beta \sin \theta=f \sin \omega t \sin \theta
$$

Where $f \sin \omega t$ is a parametric excitation that models the base excitation, ${ }^{c_{1}} \dot{\theta}$ and ${ }^{c_{2}} \dot{\theta}^{3}$ are the linear and the nonlinear damping terms, respectively, and the term $\alpha^{2}(1-\cos \theta)^{2} / \sin ^{3} \theta-\beta \sin \theta$ is the nonlinear resilience. Given the states $x_{1}=\theta, x_{2}=\dot{\theta}$, this system can be transformed into the following nominal form:

$$
\left\{\begin{array}{l}
\dot{x}_{1}=x_{2} \\
\dot{x}_{2}=-c_{1} x_{2}-c_{2} x_{2}^{3}-\alpha^{2} \frac{\left(1-\cos x_{1}\right)^{2}}{\sin ^{3} x_{1}}+(\beta+f \sin \omega t) \sin x_{1}
\end{array}\right.
$$

The nonlinear gyros system exhibits chaotic behavior for the specific parameter values of $\alpha^{2}=100, \beta=1, c_{1}=0.5, c_{2}=0.05, \omega=2, f=35.5$. The chaotic 
motion and the strange attractor of system (2) with initial conditions of $x_{1}(0)=1, x_{2}(0)=-1$ are illustrated in Fig. 1.
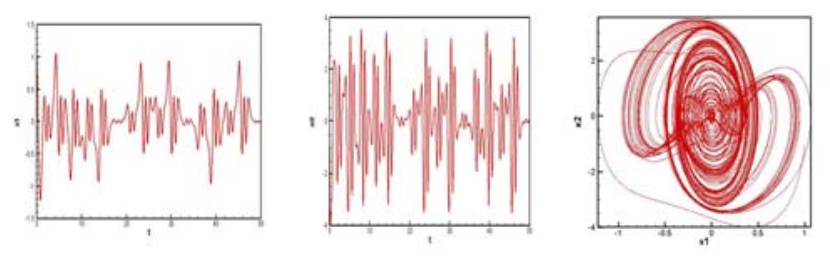

FIGURE I. STATE TRAJECTORIES OF SYSTEM AND THE CHAOTIC ATTRACTOR OF SYSTEM (1).

Assume that two above-mentioned systems are given, one is the drive system with the subscript 3 , and the other is the response system with the subscript 4 . The drive system is given by

$$
\left\{\begin{array}{l}
\dot{x}_{1}=x_{2} \\
\dot{x}_{2}=-c_{1} x_{2}-c_{2} x_{2}^{3}-\alpha^{2} \frac{\left(1-\cos x_{1}\right)^{2}}{\sin ^{3} x_{1}}+(\beta+f \sin \omega t) \sin x_{1}
\end{array}\right.
$$

The response system is given by

$$
\left\{\begin{aligned}
\dot{y}_{1}= & y_{2} \\
\dot{y}_{2}= & -c_{1} y_{2}-c_{2} y_{2}^{3}-\alpha^{2} \frac{\left(1-\cos y_{1}\right)^{2}}{\sin ^{3} y_{1}}+(\beta+f \sin \omega t) \sin y_{1} \\
& +\Delta f\left(y_{1}, y_{2}\right)+d(t)+u(t)
\end{aligned}\right.
$$

where ${ }^{x(t)=\left(x_{1}, x_{2}\right)^{\mathrm{T}}}$ is the state vector of the drive gyros, $y(t)=\left(y_{1}, y_{2}\right)^{\mathrm{T}}$ is the state vector of the response system, $\Delta f\left(y_{1}, y_{2}\right)$ and $d(t)$ are unknown model uncertainties and external disturbances of the response system, respectively; and $u(t)$ is the control input to be designed later. To solve the finite-time synchronization problem, the synchronization error between the drive (3) and response systems (4) can be defined as $e(t)=\left(e_{1}, e_{2}\right)^{\mathrm{T}}$. Let the error variables be $e_{1}=x_{2}-x_{1}, e_{2}=y_{2}-y_{1}$. Therefore, with subtracting Eq. (4) from Eq. (3), the error dynamics is obtained as follows:

$$
\left\{\begin{aligned}
\dot{e}_{1}= & e_{2} \\
\dot{e}_{2} & =-c_{1} e_{2}-\alpha^{2} \phi_{1}\left(x_{1}, y_{1}\right)-c_{2} \phi_{2}\left(x_{2}, y_{2}\right) \\
& +(\beta+f \sin \omega t) \phi_{3}\left(x_{1}, y_{1}\right)+\Delta f\left(y_{1}, y_{2}\right)+d(t)+u(t)
\end{aligned}\right.
$$

Where $\phi_{1}\left(x_{1}, y_{1}\right)=\left(1-\cos y_{1}\right)^{2} / \sin ^{3} y_{1}-\left(1-\cos x_{1}\right)^{2} / \sin ^{3} x_{1}$, $\phi_{2}\left(x_{2}, y_{2}\right)=y_{2}^{3}-x_{2}^{3} ; \phi_{3}\left(x_{1}, y_{1}\right)=\sin y_{1}-\sin x_{1}$.

Assumption 1 It is assumed that the external $^{\Delta f\left(y_{1}, y_{2}\right)}$ and disturbances $d(t)$ are norm-bounded:

$$
\left|\Delta f\left(y_{1}, y_{2}\right)\right|<h,|d(t)|<d .(h>0, d>0)
$$

Definition 1 Consider the error dynamical system (5). If there exists a constant $T=T(\boldsymbol{e}(0))>0$ such that $\lim _{t \rightarrow T}\|\boldsymbol{e}(t)\|=\lim _{t \rightarrow T}\|\boldsymbol{y}(t)-\boldsymbol{x}(t)\|=0$ and $\|\boldsymbol{e}(t)\| \equiv 0, t>T$, then states of the system (5) will converge to zero in the finite time $T$.

The control goal of this paper is to design a suitable nonsingular terminal sliding mode controller for stabilization of the uncertain system (5) around zero.

\section{IIIMAIN RESULTS}

To realize the aforementioned procedure, the terminal sliding mode can be defined

$$
s(t)=c e_{1}(t)+\left|e_{2}(t)\right|^{q / p} \operatorname{sgn} e_{2}(t)
$$

Where $c>0$, both $p$ and $q$ are positive odd integers, $1<q / p<2$.

Theorem 1 Consider the error dynamics (5), if the TSM is designed as (6), then the system state trajectory starting from any initial state $e_{1}\left(t_{r}\right) \neq 0$ will converge to zero in finite time $t_{s}$, given by $t_{s}=\frac{q}{(q-p) c^{p / q}}\left|e_{1}\left(t_{r}\right)\right|^{1-p / q}$.

Where $t_{r}$ is the time of synchronous error state trajectory reaching to the sliding surface $s(t)=0$.

Proof Consider the following Lyapunov function: $V=\frac{1}{2} e_{1}^{2}$. have

When the sliding surface $s(t)=0$ is reached, from (6), we

$$
e_{1}(t)=-\frac{1}{c}\left|e_{2}(t)\right|^{q / p} \operatorname{sgn} e_{2}(t)
$$

Then $\dot{V}=e_{1} \dot{e}_{1}=e_{1} e_{2}=-\frac{1}{c}\left|e_{2}(t)\right|^{q / p+1} \leq 0$, only when $e_{2}=0, \quad \dot{V}=0$.

From $s(t)=0$, one can obtain $e_{1}=0$. Furthermore, from (6), we have

$$
\dot{e}_{1}(t)+c^{p / q}\left(e_{1}(t)\right)^{p / q}=0
$$

Where $e_{1}(t)=0$ is the terminal attractor of the system (7).

The finite time $t_{s}$ that is taken to travel from $e_{1}\left(t_{r}\right) \neq 0$ to $e_{1}\left(t_{r}+t_{s}\right)=0$ is given by $t_{s}=-\frac{1}{c^{p / q}} \int_{e_{1}\left(t_{r}\right)}^{e_{1}\left(t_{s}+t_{r}\right)} \frac{\mathrm{d} e_{1}(t)}{\left[e_{1}(t)\right]^{p / q}}$ $=-\frac{1}{c^{p / q}} \int_{e_{1}\left(t_{r}\right)}^{0} \frac{\mathrm{d} e_{1}(t)}{\left[e_{1}(t)\right]^{p / q}}=\frac{q}{(q-p) c^{p / q}}\left|e_{1}\left(t_{r}\right)\right|^{1-p / q}$.

This completes the proof. 
Having established the suitable sliding surface, the next step is to determine an input signal $u(t)$ to guarantee that the error system trajectories reach to the sliding surface $s(t)=0$ and stay on it forever. Therefore, a sliding mode controller is proposed based on a double power reaching law [15] as:

$$
\begin{aligned}
u(t) & =c_{1} e_{2}+\alpha^{2} \phi_{1}\left(x_{1}, y_{1}\right)+c_{2} \phi_{2}\left(x_{2}, y_{2}\right) \\
& -(\beta+f \sin \omega t) \phi_{3}\left(x_{1}, y_{1}\right)-\frac{c p}{q}\left|e_{2}\right|^{2-q / p} \operatorname{sgn}\left(e_{2}\right) \\
& -(h+d) \operatorname{sgn}(s)-\left(k_{1}|s|^{\lambda}+k_{2}|s|^{\mu}\right) \operatorname{sgn}(s)
\end{aligned}
$$

Where $k_{1}>0, k_{2}>0, \lambda>1,0<\mu<1$.

Theorem 2 For system (5), if the controller is designed as (8), then system trajectories reach to the sliding surface in finite time.

Proof Consider the following Lyapunov function: $V=\frac{1}{2} s^{2}$.

Calculating the derivation along system (5) yields, from (6) and (8).

$$
\begin{gathered}
\dot{V}=s \dot{s}=s\left(c e_{2}+\frac{q}{p}\left|e_{2}\right|^{q / p-1} \dot{e}_{2}\right) \\
=s\left[c e_{2}+\frac{q}{p}\left|e_{2}\right|^{q / p-1}\left(-c_{1} e_{2}-\alpha^{2} \phi_{1}-c_{2} \phi_{2}+(\beta+f \sin \omega t) \phi_{3}\right.\right. \\
+\Delta f+d(t)+u(t))] \\
=s\left[c e_{2}+\frac{q}{p}\left|e_{2}\right|^{q / p-1}\left(-c_{1} e_{2}-\alpha^{2} \phi_{1}-c_{2} \phi_{2}+(\beta+f \sin \omega t) \phi_{3}\right.\right. \\
+\Delta f+d(t)+c_{1} e_{2}+\alpha^{2} \phi_{1}+c_{2} \phi_{2}-(\beta+f \sin \omega t) \phi_{3} \\
\left.\left.-\frac{c p}{q}\left|e_{2}\right|^{2-q / p} \operatorname{sgn}\left(e_{2}\right)-(h+d) \operatorname{sgn}(s)-\left(k_{1}|s|^{\lambda}+k_{2}|s|^{\mu}\right) \operatorname{sgn}(s)\right)\right] \\
=\frac{q}{p}\left|e_{2}\right|^{(q-p) / p} s\left[\Delta f+d(t)-(h+d) \operatorname{sgn}(s)-\left(k_{1}|s|^{\lambda}+k_{2}|s|^{\mu}\right) \operatorname{sgn}(s)\right] \\
\leq-\frac{q}{p}\left|e_{2}\right|^{(q-p) / p}\left(k_{1}|s|^{\lambda+1}+k_{2}|s|^{\mu+1}\right) \leq 0
\end{gathered}
$$$$
\text { When } e_{2} \neq 0,-\frac{q}{p}\left|e_{2}\right|^{(q-p) / p}\left(k_{1}|s|^{\lambda+1}+k_{2}|s|^{\mu+1}\right)<0 \text {, this }
$$
is $\dot{V}<0$.

Then for $e_{2}=0$, it is obtained $s(t)=c e_{1}(t)$. By substituting (8) into (5), we have

$$
\left\{\begin{array}{l}
\dot{e}_{1}=e_{2} \\
\dot{e}_{2}=\Delta f+d(t)-(h+d) \operatorname{sgn}(s)-\left(k_{1}|s|^{\lambda}+k_{2}|s|^{\mu}\right) \operatorname{sgn}(s)
\end{array}\right.
$$

So, there are $\quad \dot{e}_{2} \leq-k_{1}|s|^{\lambda}-k_{2}|s|^{\mu} \quad$ for $\quad s>0$ and $\dot{e}_{2} \geq k_{1}|s|^{\lambda}+k_{2}|s|^{\mu}$ for $s<0$

Therefore, it is concluded that the sliding mode can be reached from anywhere in the phase plane in finite time. Hence the proof is completed.

Remark 1 According to theorem 1 and theorem 2, the terminal sliding mode control law (8) and the terminal sliding surface (6) can make the response system reach the drive system in finite time.

\section{SIMULATION RESULTS}

To verify the effectiveness of the proposed finite-time controller in the synchronization of two chaotic gyros with uncertainty and external disturbance, a numerical simulation is performed. The system uncertainty and external disturbance are assumed to be $\Delta f\left(y_{1}, y_{2}\right)=-0.1 \sin \left(y_{1}\right), d(t)=0.2 \cos (\pi t)$, Respectively, The positive constants are chosen as $\alpha^{2}=100, \beta=1, \lambda=2, \quad \mu=0.5 \quad c_{1}=0.5, c_{2}=0.05$, $\omega=2, f=35.5 c=6, p=3, q=5, k_{1}=10, k_{2}=6, h=0.1, d=0.2$.

The time responses of synchronous error states are depicted in Fig. 2. We can see all the error states quickly converge to zero. As shown in Fig. 3, it is apparent that the trajectories of the response system quickly attain those of the drive system.

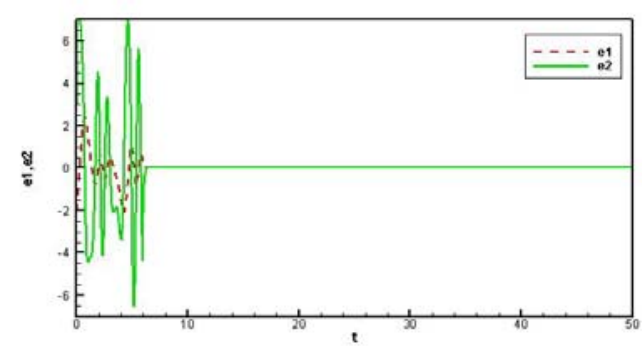

FIGURE II. SYNCHRONIZATION ERROR IN TWO CHAOTIC SYSTEMS.

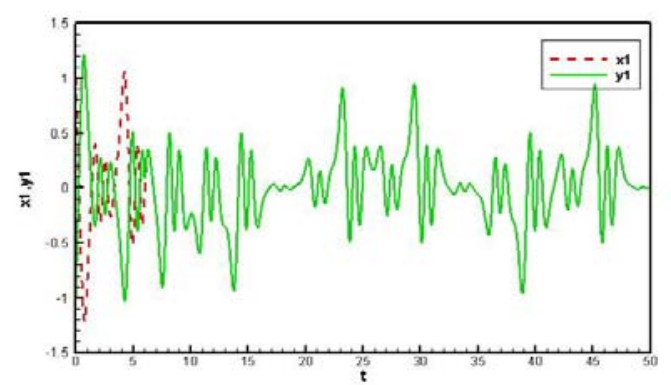




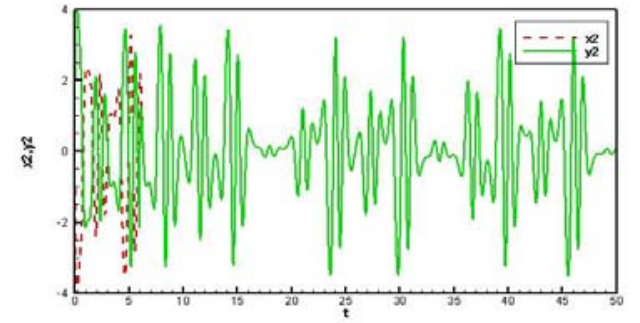

FIGURE III. STATE TRAJECTORIES OF THE DRIVE SYSTEM AND THE RESPONSE SYSTEM.

V CONCLUSIONS

In this paper, the finite-time synchronization problem between two chaotic gyros with uncertainties and external disturbances via terminal sliding mode control are considered. Based on the Lyapunov stability theorem, a sufficient finitetime synchronization criteria is derived. Finally, Numerical simulations are used to verify the proposed control techniques.

\section{ACKNOWLEDGEMENTS}

This research is supported by the National Natural Science Foundations of China (60974025).

\section{REFERENCES}

[1] Louis M. Pecora, Thomas L. Carroll. Synchronization in chaotic systems. Physical Review Letters, 64: 821-824, 1990.

[2] Steven H. Strogatz. Nonlinear Dynamics and Chaos: With Applications to Physics, Biology, Chemistry and Engineering. Perseus Books Publish, 1994

[3] ZuoLei Wang. Chaos synchronization of an energy resource system based on linear control. Nonlinear Analysis: Real world Applications, 11: 3336-3343, 2010.

[4] XinPing Guan,ZhengPing Fan, CaiLan Chen et al. Chaos Control and Application in Secure Communication. Beijing: National Defense Industry Press, 2002.

[5] Yinghui Xie, Zengqi Sun. Exponential synchronization for delayed Chen chaotic systems and applications to secure communications. Control Theory \& Applications, 27(2): 133 - 137, 2010.

[6] Haeri Mohammad, Amir Abbas Emadzadeh. Synchronizing Different Chaotic Systems using Active Sliding Mode Control. Chaos Solutions and Fractals, 31:119-129, 2007.

[7] Mohammad Pourmahmood Aghababa, Sohrab Khanmohammadi, Ghassem Alizadeh. Finite-time synchronization of two different chaotic systems with unknown parameters via sliding mode technique, Applied Mathematical Modelling, 35:3080-3091, 2011.

[8] RuiPing Xu, CunChen Gao. Synchronization of a Chaos System Based on Linear Feedback Control. Periodical of Ocean University of China, 44(5): 114-120, 2014(In Chinese).

[9] Chunlai Li, Simin Yu. A new hyperchaotic system and its adaptive tracking control. Acta Physica Sinica, 61 (4) 040504: 1-7, 2012.

[10] RuiPing $\mathrm{Xu}$, CunChen Gao. Chaos Synchronization of a Financial System Based on Linear Control. Control Engineering of China, 21(1): 18-22, 2014 (In Chinese).

[11] Chen HK. Chaos and chaos synchronization of a symmetric gyro with linear-plus-cubic damping. Journal of Sound and Vibration, 255(4):719740, 2003.

[12] Wei Xu, Hongchan Zheng, Youming Lei. Synchronization of two chaotic nonlinear gyros using active control. Physical Letters A, 343(1):153-158, 2005.
[13] JunJnh Yan, MeeiLing Hung, TehLu Liao. Adaptive sliding mode control for synchronization of chaotic gyros with fully unknown parameters. Journal of Sound and Vibration, 298: 298-306, 2006.

[14] Her-Terng Yau. Chaos synchronization of two uncertain chaotic nonlinear gyros using fuzzy sliding mode control. Mechanical Systems and Signal Processing, 22: 408-418, 2008.

[15] HeXin Zhang, JinSuo Fan, Fei Meng et al. A new double power reaching law for sliding mode control. Control and Decision, 28(2):289-293, 2013(In Chinese). 